\title{
IMPROVING INTEROPERABILITY OF GPS AND L- BAND TELEMETRY WITH SHAPED-PATTERN ANTENNAS
}

\author{
Mr. Andrew Richen \\ Mr. David Clark \\ Toyon Research Corporation
}

\author{
Mr. Stuart McNamee \\ Mr. Robert Ellington \\ Mr. Gary Johnson \\ Mr. Guy Williams \\ USAF AFFTC Edwards AFB
}

\author{
Mr. Robert Selbrede \\ JT3, Inc.
}

\begin{abstract}
In a study sponsored by the Air Force Flight Test Center at Edwards AFB, California, Toyon Research Corporation, Goleta, California, demonstrated that shaped pattern antennas could be used to mitigate interference caused by telemetry signals on GPS systems. Using a technique for fixed reception pattern antenna (FRPA) design, Toyon built and tested a GPS antenna that minimizes reception of telemetry signals from a known location.
\end{abstract}

\section{KEYWORDS}

GPS, Flight Test Instrumentation, Telemetry, L-Band, Radio Frequency Interference

\section{INTRODUCTION}

Global Positioning System (GPS) reception has become an imperative on nearly all airborne test systems. As the complexity of tests performed at test ranges increases, a growing number of signals are crowding into frequencies adjacent to the GPS band. The low power GPS signals are particularly susceptible to radio frequency interference (RFI) caused by telemetry transmitters operating on adjacent frequency bands, using antennas in close physical proximity to the GPS receive antenna. While the GPS receiver includes bandpass filters to mitigate the effect of RFI on GPS reception, the sheer disparity in 
power levels between the GPS signal and the telemetry signal creates a need for additional measures to isolate the two systems.

Toyon Research Corporation, in a study sponsored by the Air Force Flight Test Center at Edwards AFB, California, has designed and built a prototype antenna that yields an additional 25 to $30 \mathrm{~dB}$ of co- and adjacent channel isolation in addition to that provided by bandpass filtering. Our approach is to shape the frequency response and pattern of a GPS receive antenna to minimize its gain in the direction of the telemetry source for frequencies within or near the GPS frequency bands. The antenna's ability to receive GPS signals must also be maintained except at angles very near to the direction of the interference.

Our technique involves attaching carefully selected control devices to a printed antenna. These control devices effect a change on the surface currents on the antenna, and allow us to modify the electrical properties of the antenna. We have developed network analysis and optimization software to automate the process of choosing control devices that, when attached to an antenna, will yield a desired set of antenna properties. We refer to antennas developed using this design methodology as Electronically Reconfigurable Antennas (ERAs).

Under our ERA design approach, we do not view the design of the antenna element itself as the central task of the antenna design problem. Rather, we see the antenna element as a frame, with a number of connection points, or ports, where a control device or a feed line can be attached. The choice of control devices actually determines the electrical properties of the antenna. In general, the control devices can be either active or passive. In selfinterference scenarios where the power spectrum, direction, and polarization of the interfering signal are constant and known, passive control devices provide time-invariant suppression of interference.

\section{ANTENNA DESIGN AND CONSTRUCTION}

In this study, we sought to demonstrate the applicability of this technique to a particularly severe RFI scenario encountered on some aircraft when configured for flight testing. On these aircraft, structural constraints restrict the relative placement of the telemetry and GPS antennas to the configuration shown in Figure 1. In this mock-up of an aircraft panel, two telemetry blade antennas surround a single GPS receive antenna. The GPS antenna occupies a circle 4.5 inches in diameter in the center of the plate. The telemetry blade

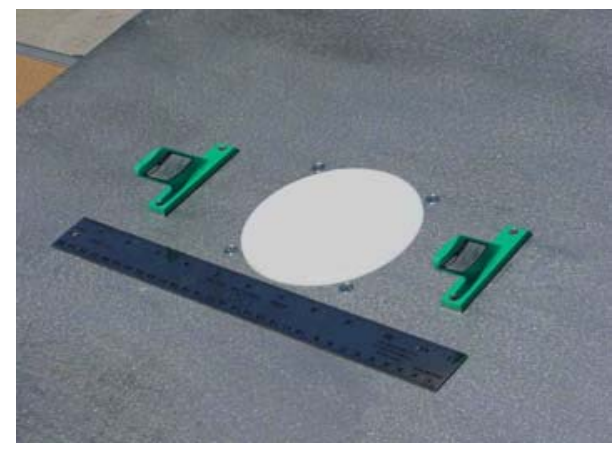

Figure 1 - GPS antenna set in aluminum housing surrounded by telemetry antennas. 
antennas are on opposite sides of the GPS antenna exactly nine inches apart from each other. The GPS antenna mounts flush with the panel surface, 0.5 inches above a recessed ground plane. The gap between the antenna and ground plane is filled by a Teflon spacer.

The RFI problem caused by this geometry is considerable, given the frequencies and power levels of the GPS and telemetry signals. The L-band telemetry transmitter used by the Air Force produces a $+37 \mathrm{dBm}$ signal with a center frequency between 1.4355 and $1.5355 \mathrm{GHz}$. This signal is run through a 1 by 2 power divider, with one output of the divider connected to each telemetry blade antenna as shown in Figure 1. The incident power to each of the two telemetry antennas is $+34 \mathrm{dBm}$. The GPS L1 P(Y) signal occupies the frequency band from 1.5654 and $1.5854 \mathrm{GHz}$, with a specified incident power level of $-133 \mathrm{dBm}$. In addition, spurious signals and phase noise from the telemetry transmitter will be present at frequencies apart from the fundamental frequency, and may reach levels as high as $-35 \mathrm{dBm}$ based on our observations.

The resulting interference levels are quite severe at frequencies both inside and outside of the GPS band. We have observed that the coupling between a standard GPS antenna and each telemetry antenna in the configuration shown in Figure 1 varies from $-20 \mathrm{~dB}$ to -35 $\mathrm{dB}$ across the 1.4355 to $1.5355 \mathrm{GHz}$ telemetry band. Based on the total transmitter power of $+37 \mathrm{dBm}$, between $+17 \mathrm{dBm}$ and $+2 \mathrm{dBm}$ of power will reach the GPS receiver, as little as $30 \mathrm{MHz}$ away from the GPS band.

We have also measured a coupling level of roughly $-20 \mathrm{~dB}$ between the standard GPS antenna and telemetry antennas within the GPS L1 band. This relatively low level of isolation means that the GPS receiver will be highly vulnerable to the transmitter's spurious outputs or phase noise that might leak into the GPS L1 band. Even if the level of these outputs is $80 \mathrm{~dB}$ below that of the telemetry carrier signal, $-46 \mathrm{dBm}$ is still delivered to each telemetry antenna, and roughly $-66 \mathrm{dBm}$ will reach the GPS receiver. This amount of power represents a jammer to signal ratio of $70 \mathrm{~dB}$ over the $-133 \mathrm{dBm} \mathrm{P}(\mathrm{Y})$ GPS signal, well above the threshold at which satellite signals can be tracked.

Toyon's method of addressing this problem is to use carefully chosen control devices to modify the frequency response and gain pattern of the GPS antenna to minimize its response to telemetry signals. The control devices are connected to coaxial cables that extend through the rear of the ground plane as shown in Figure 2. The opposite ends of these cables are soldered to the printed antenna element on the top surface of the antenna.

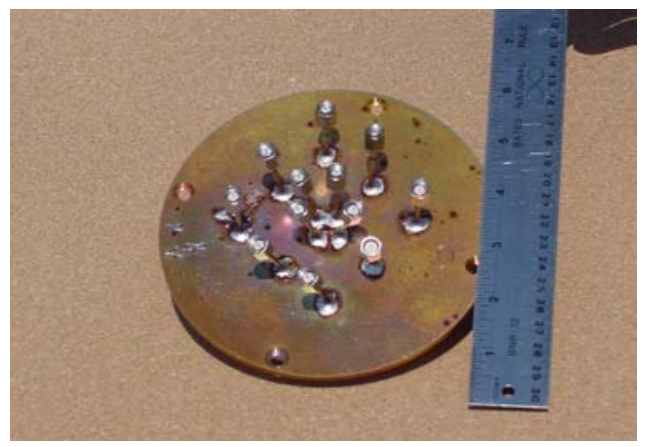

Figure 2 - Reverse side of antenna, showing ports for control devices exiting copper ground plane. 


\section{MEASURED RESULTS}

With the control devices attached to the antenna, we measured the coupling between the GPS antenna at its feed and the telemetry antennas using a network analyzer. The results of these measurements, along with coupling results using a standard GPS antenna, are shown in Figure 3. The solid trace represents the latest version of Toyon's anti-RFI antenna and the dashed trace, a commercially available GPS antenna. ${ }^{1}$ The results show that we have improved the isolation between the GPS and telemetry antennas for most frequencies within the GPS and telemetry bands. Up to $30 \mathrm{~dB}$ of improvement is seen at the upper edge of the telemetry band and at the GPS center frequency. Our antenna's performance is slightly worse than that of the standard antenna at frequencies near the lower edge of the telemetry band. Signals at these frequencies, however, are more easily rejected by the GPS receiver's bandpass filters.
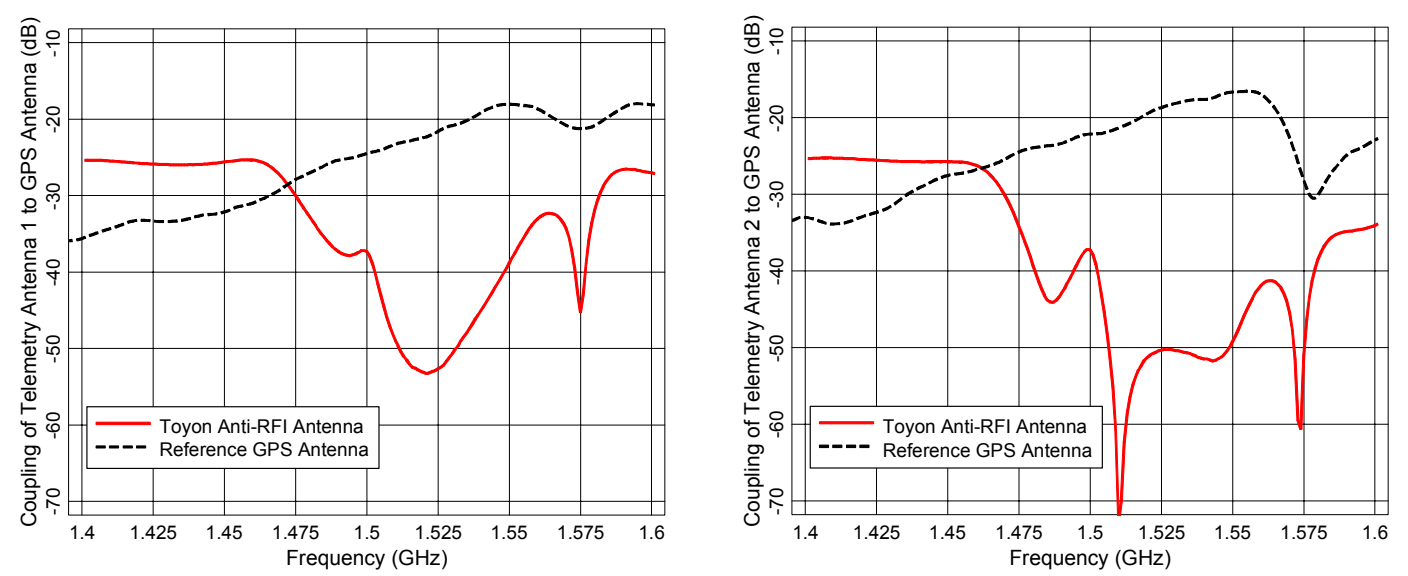

Figure 3 - Coupling to telemetry antennas for a standard GPS antenna and for Toyon's anti-RFI antenna in the configuration shown in Figure 1.

Our next step was to measure the GPS reception performance of Toyon's anti-RFI antenna compared to the reference antenna in the presence of telemetry signals. We attached the telemetry transmitter to the two blade antennas, swept the center frequency of the transmitter across the 1.4355 to $1.5355 \mathrm{GHz}$ band, and recorded the maximum GPS satellite carrier-to-noise ratio reported by the receiver. Sample measurements are tabulated in Tables 1 and 2 and complete results are plotted in Figure 4. The maximum carrier-tonoise ratio observed at the GPS receiver in its unjammed state (with the telemetry transmitter off) is roughly $50 \mathrm{~dB}-\mathrm{Hz}$.

Figure 4 shows that the reference GPS antenna is able to receive GPS signals when the telemetry transmitter is tuned over the lower portion of the telemetry band, but performance drops off rapidly as the center frequency is increased. Our antenna, in contrast, performs well over the upper two thirds of the telemetry band. Satellite signals cannot be received using our antenna when the transmitter is active and tuned to the lower quarter of the telemetry band. These results agree qualitatively with what would be predicted based on Figure 3.

1 We used the antenna supplied with Rockwell's Precision Lightweight GPS Receiver (PLGR) as a reference antenna for these experiments. 


\begin{tabular}{|c|c|c|}
\hline Frequency $(\mathrm{MHz})$ & Input Power Level $(\mathrm{dBm})$ & Maximum C/N0 $(\mathrm{dB}-\mathrm{Hz})$ \\
\hline 1440.5 & 37 & 0 \\
\hline 1460.5 & 37 & 0 \\
\hline 1480.5 & 37 & 41 \\
\hline 1500.5 & 37 & 43 \\
\hline 1520.5 & 37 & 41 \\
\hline
\end{tabular}

Table 1 - Sample results from experiment using GPS receiver and Toyon's anti-RFI GPS antenna.

\begin{tabular}{|c|c|c|}
\hline Frequency $(\mathrm{MHz})$ & Input Power Level $(\mathrm{dBm})$ & Maximum C/N0 $(\mathrm{dB}-\mathrm{Hz})$ \\
\hline 1440.5 & 37 & 49 \\
\hline 1460.5 & 37 & 32 \\
\hline 1480.5 & 37 & 0 \\
\hline 1500.5 & 37 & 0 \\
\hline 1520.5 & 37 & 0 \\
\hline
\end{tabular}

Table 2 - Sample results from experiment using GPS receiver and reference GPS antenna.

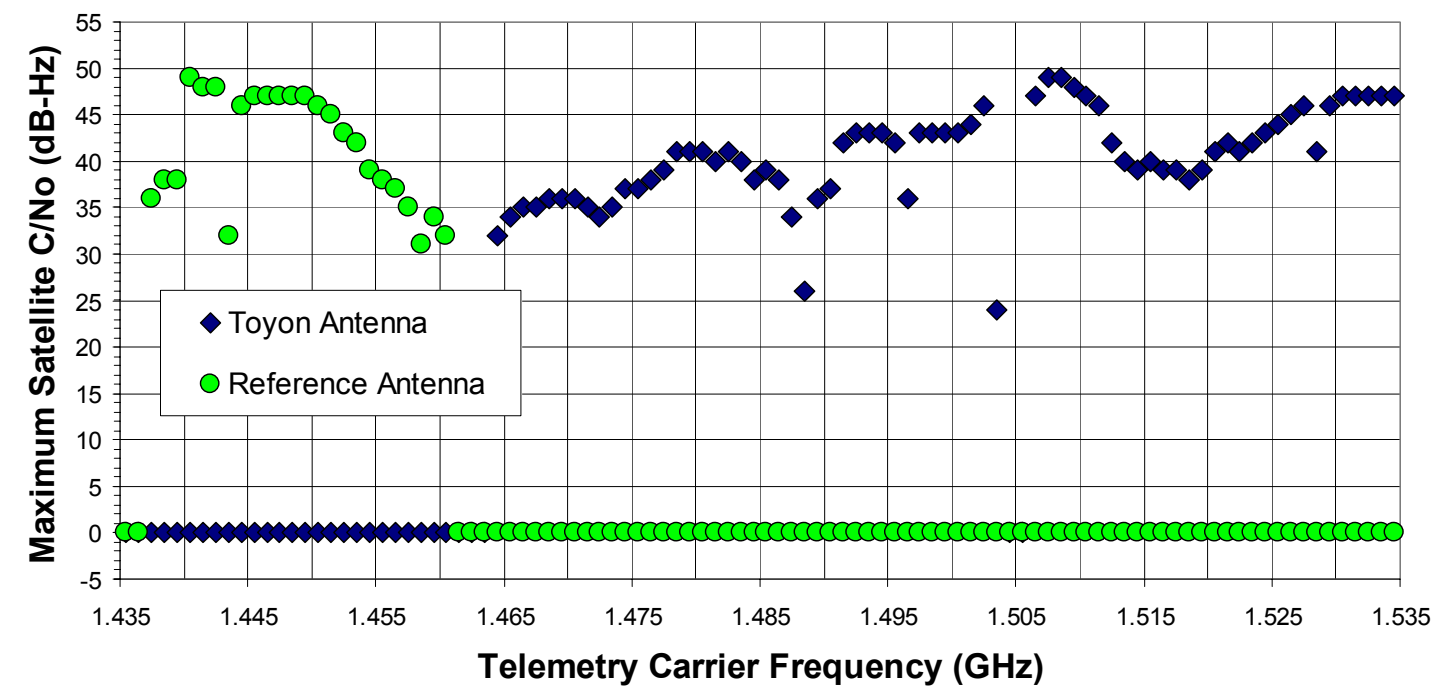

Figure 4 - Maximum GPS satellite carrier-to-noise ratio plotted vs. telemetry transmitter center frequency for Toyon's anti-RFI antenna and the reference antenna. A value of zero indicates that no satellites could be tracked when the transmitter was set to that frequency.

Neither antenna, in this case, allows for operation of GPS as the transmitter is swept over the entire frequency band. Our antenna, however, performs better when the telemetry center frequency is close to the GPS L1 center frequency, and worse as the telemetry frequency is moved further away. This capability can be complemented by adding an additional bandpass filter between the antenna and the GPS receiver, which attenuates frequencies close to its passband only slightly, but isolates well at frequencies farther away. In contrast, use of a filter in tandem with the standard antenna appears unlikely to improve the overall performance of the system, as neither filter nor antenna can provide 
good isolation at frequencies close to the GPS L1 band. We repeated the experiment whose results are shown in Figure 4 using the GPS L1 chip filter depicted in Figure 5.

The frequency response of the filter is shown in Figure 6. This is not the ideal filter for this application, because the passband insertion loss is $3 \mathrm{~dB}$. The filter is placed between the antenna and the receiver, so its insertion loss decreases the signal strength of all of the GPS satellites by 3 dB. Despite this drawback, experiments performed using the RF filter illustrate its benefit in augmenting Toyon's antenna technology.

Measurements of the combined GPS reception capability of the receiver, antenna, and filter as the transmitter is swept across several frequencies in the telemetry band are shown in Tables 3 and 4. Complete results are shown in Figure 7. The Toyon antenna with filter attached is able to track satellites with the telemetry transmitter tuned to every frequency within the $1435 \mathrm{MHz}$ to $1535 \mathrm{MHz}$ band. The relative performance of Toyon's anti-RFI antenna with and without the filter shows that while the passband insertion loss of the filter degrades the performance of the receiver by 2 $-4 \mathrm{~dB}$ at the upper end of the band, isolation is considerably improved at the lower end of the telemetry band.

The performance of the reference antenna is only slightly improved through the use of the filter. This is the expected result, even though the attenuation of the filter (Figure 6) is quite good at $1.525 \mathrm{GHz}$ and below. We hypothesize that as the telemetry center frequency is raised into the upper half of the band, the telemetry transmitter's spurious signals and phase noise occurring in the GPS band become as much of an interference problem as the telemetry signal itself. These signals are in the passband of both the external filter of Figure 5 and the GPS receiver's internal filters. The coupling profiles of Figure 3 show that Toyon's antenna is 25 to $30 \mathrm{~dB}$ better than the standard antenna at rejecting signals from the telemetry antennas occurring at $1.575 \mathrm{GHz}$. Toyon's anti-RFI GPS antenna is most effective at frequencies where the filter is least effective, and vice versa. 
Frequency $(\mathrm{MHz})$

Input Power Level (dBm)

Maximum C/N0 (dB-Hz)

\begin{tabular}{|c|c|c|}
\hline 1440.5 & 37 & 48 \\
\hline 1460.5 & 37 & 47 \\
\hline 1480.5 & 37 & 48 \\
\hline 1500.5 & 37 & 47 \\
\hline 1520.5 & 37 & 45 \\
\hline
\end{tabular}

Table 3 - Sample results from experiment using GPS receiver and Toyon's anti-RFI GPS antenna with filter attached.

Frequency $(\mathrm{MHz})$ Input Power Level (dBm)

Maximum C/N0 (dB-Hz)

\begin{tabular}{|c|c|c|}
\hline 1440.5 & 37 & 47 \\
\hline 1460.5 & 37 & 36 \\
\hline 1480.5 & 37 & 44 \\
\hline 1500.5 & 37 & 0 \\
\hline 1520.5 & 37 & 0 \\
\hline
\end{tabular}

Table 4 - Sample results from experiment using GPS receiver and reference GPS antenna with filter attached.

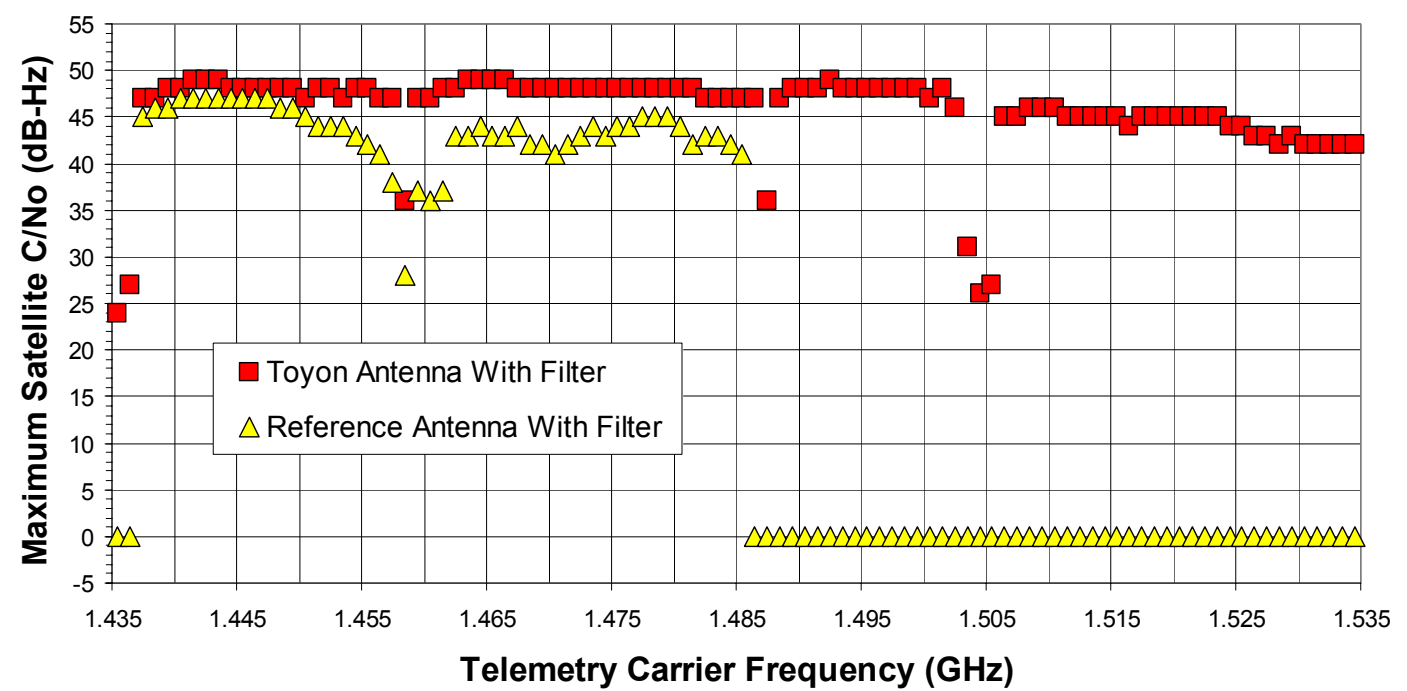

Figure 7 - Maximum GPS satellite carrier-to-noise ratio plotted vs. telemetry transmitter center frequency for Toyon's anti-RFI antenna and the reference antenna with identical filters placed between the antenna and the GPS receiver. A value of zero indicates that no satellites could be tracked when the transmitter was set to that frequency.

\section{CONCLUSION}

In our work so far on this program, we have optimized the design of our antenna to respond to a single, very severe RFI scenario. By using our Electronically Reconfigurable Antenna (ERA) technique, we designed an antenna to mitigate the effect of radio frequency interference due to nearby telemetry transmitters. We found that use of our antenna allowed simultaneous operation of the telemetry transmitter and GPS receiver when the transmitter was tuned to most, but not all, frequencies within the 1.4355 to 
$1.5355 \mathrm{GHz}$ telemetry band. When we used our antenna in tandem with a bandpass filter, we could set the telemetry transmitter to any frequency within the 1.4355 to $1.5355 \mathrm{GHz}$ band while still tracking GPS satellites.

Because of the proximity of the telemetry transmitters to the GPS antenna, it was necessary to focus most of our attention in mitigating the coupling between antennas. The gain and phase patterns of the antenna for GPS reception were secondary concerns during this early stage, but must be addressed before this antenna can be used in flight testing. The bandwidth of the isolation in the GPS L1 band and performance at L2 are other areas where work still needs to be done. The work presented in this paper represents the feasibility study portion of this program. The remainder of this ongoing work will focus on transitioning this technology into a broadly applicable tool for mitigating RFI on flight test platforms. 